\title{
Characteristics of $d r 1790$ disruptant and its functional analysis in Deinococcus radiodurans
}

\author{
Jianhui Cheng ${ }^{1}, \mathrm{Hu} \mathrm{Wang}^{3}, \mathrm{Xin}_{\mathrm{Xu}}{ }^{2}$, Liangyan $\mathrm{Wang}^{2}$, Bing Tian ${ }^{2}$, Yuejin Hua ${ }^{2}$ \\ ${ }^{1}$ Institute of Horticulture, Zhejiang Academy of Agricultural Sciences, Hangzhou, China. \\ ${ }^{2}$ Institute of Nuclear-Agricultural Sciences, Zhejiang University, Hangzhou, China. \\ ${ }^{3}$ Institute of Ageing Research, Hangzhou Normal University, Hangzhou, China.
}

Submitted: May 3, 2013; Approved: December 19, 2014.

\begin{abstract}
Deinococcus radiodurans (DR) is an extremophile that is well known for its resistance to radiation, oxidants and desiccation. The gene $d r 1790$ of $D$. radiodurans was predicted to encode a yellow-related protein. The primary objective of the present study was to characterize the biological function of the DR1790 protein, which is a member of the ancient yellow/major royal jelly (MRJ) protein family, in prokaryotes. Fluorescence labeling demonstrated that the yellow-related protein encoded by $d r 1790$ is a membrane protein. The deletion of the $d r 1790$ gene decreased the cell growth rate and sensitivity to hydrogen peroxide and radiation and increased the membrane permeability of D. radiodurans. Transcript profiling by microarray and RT-PCR analyses of the $d r 1790$ deletion mutant suggested that some genes that are involved in protein secretion and transport were strongly suppressed, while other genes that are involved in protein quality control, such as chaperones and proteases, were induced. In addition, the expression of genes with predicted functions that are involved in antioxidant systems, electron transport, and energy metabolism was significantly altered through the disruption of $d r 1790$. Moreover, the results of proteomic analyses using 2-DE and MS also demonstrated that DR1790 contributed to D. radiodurans survival. Taken together, these results indicate that the DR1790 protein from the ancient yellow protein family plays a pleiotropic role in the survival of prokaryotic cells and contributes to the extraordinary resistance of $D$. radiodurans against oxidative and radiation stresses.
\end{abstract}

Key words: Deinococcus radiodurans, $d r 1790$ disruptant, characteristics, functional analysis.

\section{Introduction}

D. radiodurans exhibits resistance to the lethal and mutagenic effects of DNA damaging agents, including $\gamma$-ray and UV radiation, hydrogen peroxide and desiccation (Battista, 1997; Makarova et al., 2001; Shu and Tian, 2010; Ghosal et al., 2005). These bacteria can survive $\sim 12 \mathrm{kGy}$ $\gamma$-ray irradiation, which generates approximately 200 double-strand and 3000 single-strand breaks per genome (Battista, 2000). The robustness of this bacterium reflects strong oxidative stress resistance mechanisms that protect proteins from oxidative damage (Wang et al., 1995; Markillie et al., 1999; Daly et al., 2007) and a DNA repair process that efficiently and precisely reassembles DNA fragments (Minton 1994; Slade et al., 2009). Antioxidant protection and repair mechanisms for DNA and other proteins enable these molecules to retain their catalytic activity and to provide a swift response under oxidative stress conditions (Slade and Radman, 2011). Genetic engineering techniques may be applied to D. radiodurans, which has extreme resistance, as well as the ability to self-repair DNA damage, to bioremediate radioactive waste sites, to breed plants for resistance and to treat human cancer. Therefore, D. radiodurans, which is of interest to many researchers, represents a microbial resource with great development prospects. D. radiodurans strains that express the cloned $\mathrm{Hg}(\mathrm{II})$ resistance gene (merA) from the $E$. coli strain BL308 exhibit growth in the presence of both $60 \mathrm{~Gy} / \mathrm{h}$ of ${ }^{137} \mathrm{Cs}$ radiation (a dose rate that exceeds those in most radioactive 
waste sites) and 30-50 $\mu \mathrm{M} \mathrm{Hg}(\mathrm{II})$ and that effectively reduce $\mathrm{Hg}(\mathrm{II})$ to the less toxic volatile elemental $\mathrm{Hg}(0)$ (Brim et al., 2000). The cloning of toluene dioxygenase tod genes from Pseudomonas putida $\mathrm{F} 1$ into the chromosome of $D$. radiodurans conferred the ability to oxidize toluene, chlorobenzene, 3,4-dichloro-1-butene, and indole in a highly irradiating environment (Lange et al., 1998). The expression of $\operatorname{Ir} E$, which is a global regulator for extreme radiation resistance in $D$. radiodurans, significantly enhanced salt tolerance in Brassica napus plants. Transgenic B. napus plants that express the $\operatorname{IrrE}$ can tolerate $350 \mathrm{mM} \mathrm{NaCl}$, which is a concentration that inhibits the growth of almost all crop plants (Pan et al., 2009). The human bone marrow cell line KG1a, which was transformed with $d r 1709$ from $D$. radiodurans, exhibited a much higher survival fraction than the original KG1a cells when treated with $\gamma$-ray radiation (Shu and Tian, 2012). However, the underlying mechanisms of $D$. radiodurans resistance to stresses remain unclear. Therefore, the identification and functional analysis of new genes that are associated with anti-radiation, DNA repair and antioxidants will improve our understanding of the extreme radiation resistance mechanisms of this strain and provide strategies for research regarding the radiation damage defense and oxidative stress resistance systems of organisms.

dr1790, which is a gene that encodes a putative yellow-related protein homolog, was identified in the $D$. radiodurans genome (Makarova et al., 2001). Interestingly, this yellow-related protein is typically detected in insects and plays important roles in pigmentation and insect behaviors. The deletion of the yellow protein gene locus in Drosophila not only affects larval pigmentation but also appears to affect insect behavior (Maleszka and Kucharski, 2000; Drapeau et al., 2006). Yellow protein can be secreted from cells because this protein contains a secretion signal peptide (Drapeau, 2003). Furthermore, other members of the Yellow/Major Royal Jelly (MRJ) protein family are expressed in not only insects but also some bacterial and fungi species, suggesting that yellow proteins are evolutionarily ancient (Drapeau et al., 2006). Although a few studies have demonstrated an association between melanization and behavior in Drosophila, and a unique clade of genes from Apis mellifera may be involved in caste specification, the function of most yellow protein family members remains largely unknown (Ferguson et al., 2011). Currently, no studies concerning the function of yellow-related proteins in prokaryotes exist. DR1790 expression was induced in a D. radiodurans mutant strain that was deficient in OxyR, which is a peroxide sensor and transcription regulator that senses the presence of reactive oxygen species and that induces the antioxidant system of $D$. radiodurans (Chen et al., 2008). These findings prompted us to investigate the functions of this yellow-related protein homolog in this extremophilic bacterium.

\section{Materials and Methods}

\section{Bacterial strains and materials}

All D. radiodurans cultures were grown at $30{ }^{\circ} \mathrm{C}$ in tryptone-yeast extract-glucose (TGY) media $(0.5 \%$ bactotryptone, $0.3 \%$ bacto-yeast extract, and $0.1 \%$ glucose) with aeration or on TGY plates solidified with $1.5 \%$ agar. Overnight cultures were incubated in fresh TGY medium, and exponential-phase cells $\left(\mathrm{OD}_{600 \mathrm{~nm}}=0.8\right)$ were used for all experiments. The E. coli strain JM109 was grown in LuriaBertani (LB) broth (1.0\% bacto-tryptone, $0.5 \%$ bacto-yeast extract, and $1.0 \% \mathrm{NaCl}$ ) or on LB plates solidified with $1.5 \%$ agar at $37^{\circ} \mathrm{C}$.

\section{Construction of mutant strains}

The $D$. radiodurans strain $\mathrm{R} 1 \Delta d r 1790$ was constructed using a deletion replacement method as described previously (Xu et al., 2008). The primers that were used in this study are listed in Table 1 . The primers $\mathrm{p} 1$ and $\mathrm{p} 2$ were used to amplify a Bam HI fragment upstream of the targeted genes, and the primers $\mathrm{p} 3$ and $\mathrm{p} 4$ were used to amplify a HindIII fragment downstream of the targeted genes. The kanamycin resistance cassette containing the GroEL promoter was obtained from the pRADK shuttle plasmid (Gao et al., 2005). The three DNA fragments were digested and ligated; then, the ligation products were used as templates for PCR $\left(30\right.$ cycles at $94{ }^{\circ} \mathrm{C}$ for $1 \mathrm{~min}, 55^{\circ} \mathrm{C}$ for $45 \mathrm{~s}$, and 72 ${ }^{\circ} \mathrm{C}$ for $1 \mathrm{~min}$ ) with $\mathrm{p} 1$ and $\mathrm{p} 4$. The resulting PCR fragments were transformed into $D$. radiodurans cells using the $\mathrm{CaCl}_{2}$ technique, and the mutant strains were selected on TGY agar plates supplemented with $20 \mu \mathrm{g} / \mathrm{mL}$ kanamycin.

\section{Complementation of R1 $1 \Delta d r 1790$}

The complementation plasmid was constructed as described previously (Gao et al., 2005; Wu et al., 2009). Briefly, chromosomal DNA was isolated from wild type strains. The 1167-bp region containing the $d r 1790$ gene was PCR-amplified $\left(35\right.$ cycles at $94^{\circ} \mathrm{C}$ for $1 \mathrm{~min}, 58^{\circ} \mathrm{C}$ for $50 \mathrm{~s}$ and $72^{\circ} \mathrm{C}$ for $1 \mathrm{~min}$ ) using the primers DR1790 com $\mathrm{F}$ and DR1790 ${ }_{\text {com }} \mathrm{R}$ (Table 1) and ligated into the pMD18 T-Easy vector (Takara, JP); the resulting construct was designated as pMD- $d r 1790$. After digestion with $N d e I$ and BamHI, the target gene $d r 1790$ was ligated into NdeI- and BamHI-pre-digested pRADK, and the resulting construct was designated as pRAD- $d r 1790$. The complementation plasmids were confirmed by PCR and DNA sequence analyses; thus, transformation into R1 $\Delta d r 1790$ generated the functional complementation strain mutant Dr1790com.

\section{Measurement of growth rate}

The growth rate was measured as described previously (Mattimore et al., 1995). Briefly, $500 \mu \mathrm{L}$ overnight culture of each strain was transferred to $50 \mathrm{~mL}$ TGY medium. The culture was grown at $30{ }^{\circ} \mathrm{C}$ with agitation 
(200 rpm). Then, the culture dilutions were spread onto TGY agar plates after $2\left(t_{1}\right)$ and $4 \mathrm{~h}\left(\mathrm{t}_{2}\right)$. The plates were incubated at $30^{\circ} \mathrm{C}$ for 5 days, and subsequently, the number of colony-forming units (CFU) was determined. The dou- ble time $(g)$ was calculated according to the following formula: $g=\ln 2 /\left(\left(\log _{10} \mathrm{~N}_{2}-\log _{10} \mathrm{~N}_{1}\right) 2.303 / \Delta \mathrm{t}\right)$, where $\mathrm{N}_{1}$ is CFU per milliliter at $t_{1}$, and $\mathrm{N}_{2}$ is CFU per milliliter at $\mathrm{t}_{2}$.

Table 1 - Primers used in this study.

\begin{tabular}{|c|c|}
\hline Primer & Sequence \\
\hline \multicolumn{2}{|c|}{ Construction of the R1 $\Delta d r 1790$ mutant } \\
\hline p1 & 5' GGTGTGTTTGACTGAGGCCGAGGAC 3' \\
\hline p2 & 5' GTTGGATCCCAGGGGTATAAGACGC 3, \\
\hline p3 & 5' TTTAAGCTTGCTGCACGTTGACCCT 3' \\
\hline $\mathrm{p} 4$ & 5' TGTTGTGTTGCCTACCTGGCGATTG 3' \\
\hline Kanamycin F & 5' CACACAGGAAACAGCTATGACCATGATTA 3' \\
\hline Kanamycin R & 5' ACAGACGGATCCTAGAAAAACTCATCGAGCATC 3' \\
\hline \multicolumn{2}{|c|}{ Complementation of the R $1 \Delta d r 1790$ mutant } \\
\hline DR1790 com F & 5' TTTCATATGATGAAAATCAAGCTGACCGC 3' \\
\hline $\mathrm{DR} 1790_{\text {com }} \mathrm{R}$ & 5' TTTGGATCCTTATTTCAGCAGCACCGGC 3' \\
\hline \multicolumn{2}{|c|}{ Real-time quantitative PCR } \\
\hline \multirow[t]{2}{*}{ DR0089 } & F: 5' TACCGCTCTTACCCCGACTC 3' \\
\hline & R: 5' CGTGTAGATGGCGAACACCA 3' \\
\hline \multirow[t]{2}{*}{ DR0126 } & F: 5' TGACGACTACGGTGGATGTGC 3', \\
\hline & R: 5' CTCGTCGCTGAGGTCTTTGG 3' \\
\hline \multirow[t]{2}{*}{ DR0128 } & F: 5' GCAACCGCACCACCATCG 3' \\
\hline & R: 5' TTCGTCTTCGTCACCAGCAAC 3' \\
\hline \multirow[t]{2}{*}{ DR0129 } & F: 5' CGCAAGGGCAACGAAACTG 3' \\
\hline & R: 5' GGTGATGAAGGGCAGGGAGAT 3' \\
\hline \multirow[t]{2}{*}{ DR0194 } & F: 5' CTCACCGACCACTACGACCCG 3' \\
\hline & R: 5' CGCCCCGCCGAACAGAAT 3' \\
\hline \multirow[t]{2}{*}{ DR0350 } & F: 5' CAGATAGCCACGCTCAACGC 3', \\
\hline & R: 5' CGACCCGGAAGCCCTTTT 3' \\
\hline \multirow[t]{2}{*}{ DR0606 } & F: 5' CGAAGAAGCCGAGCAGAAGA 3, \\
\hline & R: 5’ GGTGCCGTTGTCCAGGGTC 3’' \\
\hline \multirow[t]{2}{*}{ DR0607 } & F: 5' AGCACCGACTCCGACTACGC 3' \\
\hline & R: 5’ GCCTGCCACGATGCCTTCT 3' \\
\hline \multirow[t]{2}{*}{ DR0888 } & F: 5' AGGTGACGGGTGAGGTGGC 3', \\
\hline & R: 5' GCTGGGGCTGGTTTGTGC 3' \\
\hline \multirow[t]{2}{*}{ DR1046 } & F: 5' CGGCGACAGTTTCGTGGC 3', \\
\hline & R: 5' GCTGTTCACTGGTTTTGTTGGTC 3', \\
\hline \multirow[t]{2}{*}{ DR1114 } & F: 5' CCCCGAACTTCACTCCCA 3' \\
\hline & R: 5' CGGTCAGGGTCTGGTTTTCA 3' \\
\hline \multirow[t]{2}{*}{ DR1148 } & F: 5' CATATGGTTTTTCATGGACGGCTCC3' \\
\hline & R: 5' GGATCCTCAAGAGTCGGCCCCGCTA3' \\
\hline \multirow[t]{2}{*}{ DR1172 } & F: 5' GTCTGTTGCTGCTCGGTGCC 3' \\
\hline & R: 5' TGGTCTTTTCCCAGCCCTTG 3' \\
\hline \multirow[t]{2}{*}{ DR1909 } & F: 5' GCCTACACGCACGTTTCCG 3' \\
\hline & R: 5' CCTCACGCACCACGCAGA 3' \\
\hline \multirow[t]{2}{*}{ DR1974 } & F: 5' GCCACCTGGACCCCTGAG 3' \\
\hline & R: 5' GCATTCCGGCTTCTTCGAT 3' \\
\hline
\end{tabular}




\section{Cell survival under oxidative stress and ionizing radiation}

The hydrogen peroxide sensitivity of $D$. radiodurans cells was assayed as described previously (Wang et al., 2008), with some modifications. The cells were harvested during the early stationary phase $\left(\mathrm{OD}_{600 \mathrm{~nm}}=1.0\right)$, washed twice and re-suspended with phosphate buffer $(20 \mathrm{mM}, \mathrm{pH}$ 7.4). An aliquot was removed as a control, and the remaining suspension was treated with hydrogen peroxide at a final concentration of $20 \mathrm{mM}$. The mixture was incubated at $30{ }^{\circ} \mathrm{C}$ in an orbital shaker. Catalase (Sigma-Aldrich) was added in excess ( $15 \mathrm{U})$ to terminate the $\mathrm{H}_{2} \mathrm{O}_{2}$ treatment. Then, the cells were diluted and spread onto solid TGY media to determine the number of CFUs. The survival fractions were defined as a percentage of the CFU obtained in the treated sample compared with the control. The data are presented as the means $\pm \mathrm{SD}$ of three independent experiments.

The cell survival fractions under ionizing radiation were determined using a previously described method (Wang et al., 2008).

\section{Measurement of protein carbonylation levels}

Protein carbonylation, which is an indicator of intracellular protein oxidation, was measured using the DNPH (2,4-dinitrophenyl hydrazine) method (Tian et al., 2009).

\section{Membrane localization of the DR1790 protein}

The plasmid pRADG- $d r 1790$ was constructed as described previously (Gao et al., 2008). pRADG- $d r 1790$ was transformed into the R1 $\Delta d r 1790$ mutant strain. The transformant was obtained by chloramphenicol-resistance selection. The transformant was grown to the exponential phase $\left(\mathrm{OD}_{600 \mathrm{~nm}}\right.$ is approximately 0.8$)$, spread on a glass slide and examined using a laser confocal microscope (Zeiss LSM510, Germany).

\section{Membrane integrity assessment}

Differences in membrane permeability between the varying strains were assessed using a LIVE/DEAD BacLight Bacterial Viability Kit (Invitrogen, Carlsbad, CA, USA). This system employs two nucleic acid stains: green-fluorescent SYTO9 stain and red-fluorescent propidium iodide (PI) stain. Live cells with intact membranes fluoresced green, while dead cells or cells with compromised membranes fluoresced red. Bacterial cells were grown to mid-exponential phase, and a 1-mL aliquot of the culture was normalized to an $\mathrm{OD}_{600 \mathrm{~nm}}$ equal to 0.6 , washed twice with PBS, and resuspended in $1 \mathrm{~mL}$ PBS. The bacterial suspensions were stained with the nucleic acid dyes according to the manufacturer's protocol; then, $10 \mu \mathrm{L}$ stained bacteria was spotted onto glass coverslips and visualized using a Leica DM4000B wide-field epifluorescence microscope (Leica Microsystems, Wetzlar, Germany). In total,
10 different fields were viewed for each strain, and the numbers of green, red or mixed cells were counted for each field.

\section{Transcriptome analysis}

The procedures used for microarray hybridization and data analyses were performed as described previously (Chen et al., 2008). Briefly, total RNA was prepared from three replicates of wild type and R1 $\Delta d r 1790$ mutant cells. Approximately $16 \mu \mathrm{g}$ total RNA was annealed with $10 \mu \mathrm{g}$ random hexamer primers in a total volume of $20 \mu \mathrm{L}$ at $70{ }^{\circ} \mathrm{C}$ for $10 \mathrm{~min}$, followed by incubation on ice for $2 \mathrm{~min}$. cDNA synthesis was performed at $42{ }^{\circ} \mathrm{C}$ overnight in a $31-\mu \mathrm{L}$ reaction mixture using SuperScript III Reverse Transcriptase (Invitrogen) with $0.5 \mathrm{mM}$ dNTP mix containing amino allyl-dUTP (GE, Piscataway, NJ, USA). The reaction was terminated by adding $20 \mu \mathrm{L} 0.5 \mathrm{M}$ EDTA and $20 \mu \mathrm{L} 1 \mathrm{M} \mathrm{NaOH}$, followed by heating at $65^{\circ} \mathrm{C}$ for $20 \mathrm{~min}$. The reaction mixture was neutralized with $50 \mu \mathrm{L} 1 \mathrm{M}$ HEPES buffer ( $\mathrm{pH} 7.0$ ), and unincorporated amino allyl-dUTPs were removed by ultra-filtration using YM 30 columns (Millipore). The cDNA was coupled to 1 pmol $\mathrm{Cy} 3$ or Cy 5 dye (GE) in $0.1 \mathrm{M}$ sodium carbonate buffer for $2 \mathrm{~h}$ at room temperature, and free $\mathrm{Cy} 3$ or $\mathrm{Cy} 5$ was removed. The labeled pools of wild type and R1 $\Delta d r 1790$ mutant cDNA were mixed and simultaneously hybridized with the DNA chips in a solution containing $3 \mathrm{X}$ saline sodium citrate (SSC), $0.3 \%$ SDS, and $24 \mu \mathrm{g}$ unlabeled herring sperm DNA (Gibco BRL, Gaithersburg, MD, USA). Normalization and statistical analysis were performed in the R computing environment (version 2.2.0, $\mathrm{R}$ with Aqua for Windows) using the linear modeling features of the Limma microarray data package (Wettenhall et al., 2004). Before channel normalization, microarray outputs were filtered using Limma to remove spots with poor signal quality by excluding data points with a mean intensity less than two standard deviations above the background in both channels. Then, global LOESS normalization was used to normalize all data, and three replicate spots per gene in each array were used to maximize the robustness of the differential expression measurement of each gene via the "ImFit" function. The transcriptome analysis data were deposited in the Gene Expression Omnibus database under accession no. GSE22628.

\section{Real-time quantitative PCR}

The genes of interest were identified using real-time quantitative PCR to validate the results of the microarray data. dr0089, which is a gene whose expression is unaffected by $\mathrm{H}_{2} \mathrm{O}_{2}$ and ionizing radiation, was used for normalization. Briefly, first-strand cDNA synthesis was performed in $20-\mu \mathrm{L}$ reactions containing $1 \mu \mathrm{g}$ each DNase I-treated and purified total RNA sample and $3 \mu \mathrm{g}$ random hexamers. The real-time PCR amplification was performed 
using a Toyobo SYBR Green I Real Time PCR kit (Japan) according to the manufacturer's instructions under the following conditions: $94^{\circ} \mathrm{C}$ for $2 \mathrm{~min}$, followed by 40 cycles at $94{ }^{\circ} \mathrm{C}$ for $10 \mathrm{~s}$, annealing at $56-62^{\circ} \mathrm{C}$ for $15 \mathrm{~s}$ and $72{ }^{\circ} \mathrm{C}$ for $30 \mathrm{~s}$. All assays were performed using a Stratagene Mx3005P qPCR system (Stratagene, Cedar Creek, TX, USA).

\section{Proteomics analysis}

Proteomic analysis of the mutant compared with the wild type strain was performed using 2-DE and data analyses, in-gel digestion, MALDI-TOF MS analysis and a PMF spectra-based database search (Lu et al., 2009).

\section{Results}

\section{Growth of the deletion mutant}

We assayed the doubling time of cells in the lag and $\log$ phases. The R1 $\Delta d r 1790$ mutant doubling time $(2.1 \pm 0.4 \mathrm{~h})$ was not significantly slower than the doubling time $(1.5 \pm 0.4 \mathrm{~h})$ of the wild type $\mathrm{R} 1$ strain under aerobic conditions during the lag phase $(p>0.05)$ (Figure 1A). However, the R1 $\Delta d r 1790$ mutant doubling time $(3.1 \pm 0.5 \mathrm{~h})$ was slightly slower than the doubling time $(1.6 \pm 0.2 \mathrm{~h})$ of the wild type R1 strain under aerobic conditions during the log phase $(p<0.05)$ (Figure 1B).

The deletion mutant was sensitive to oxidative stress and radiation

The yellow-related protein homolog DR1790 from $D$. radiodurans functions has been implicated in cell resistance to oxidative stress and radiation. The R1 $\Delta d r 1790 \mathrm{mu}-$ tant was sensitive to hydrogen peroxide treatment and $\gamma$-ray radiation. Compared with the wild type strains, the survival of the R1 $\Delta d r 1790$ mutant cells decreased nearly 15 -fold in response to $30 \mathrm{mM}$ hydrogen peroxide (Figure $2 \mathrm{~A}$ ) and nearly 3 -fold in response to a $8 \mathrm{kGy}$ dose (Figure 2B).

To determine whether the loss of DNA damage tolerance in the R1 $\Delta d r 1790$ mutant reflected the absence of $d r 1790$ and not a polar effect of this mutation, the wild type allele of this gene was cloned into pRADgro, which is a Deinococcus expression vector, and the protein was expressed in R1 $\Delta d r 1790$ mutant cells. The radiation and oxidative resistance of the complemented mutant Dr1790com strain nearly recovered to the phenotype of the wild type strain (Figure 2), suggesting that the sensitivity of the R1 $\Delta d r 1790$ mutant reflected the absence of the $d r 1790$ gene.

\section{Comparison of intracellular protein oxidation levels between the wild type R1 strain and the R1 $\mathrm{d} d r 1790$ mutant strain}

The level of protein oxidation in the R $1 \Delta d r 1790 \mathrm{mu}-$ tant was analyzed and compared with that of the wild type R1 strain (Figure 3). The total protein carbonyl contents measured in the wild type and mutant strains were 0.012 and $0.015 \mathrm{mmol} / \mathrm{mg}$, respectively, indicating that the mutant strain exhibited relatively higher levels of protein oxidation compared with the wild type strain in the absence of oxygen stress. Following $\mathrm{H}_{2} \mathrm{O}_{2}$ treatment, intracellular protein carbonylation significantly increased in both the wild type and R1 $\Delta d r 1790$ mutant strains. The carbonyl content in the $\mathrm{R} 1 \Delta d r 1790$ mutant post- $\mathrm{H}_{2} \mathrm{O}_{2}$ treatment was $0.023 \mathrm{mmol} / \mathrm{mg}$ protein, which was significantly higher than the content in the wild type cells $(0.017 \mathrm{mmol} / \mathrm{mg}$ protein, $p<0.05)$, suggesting that the intracellular proteins in the mutant cells lacking DR1790 were more sensitive to oxidative damage than those in the wild type cells.
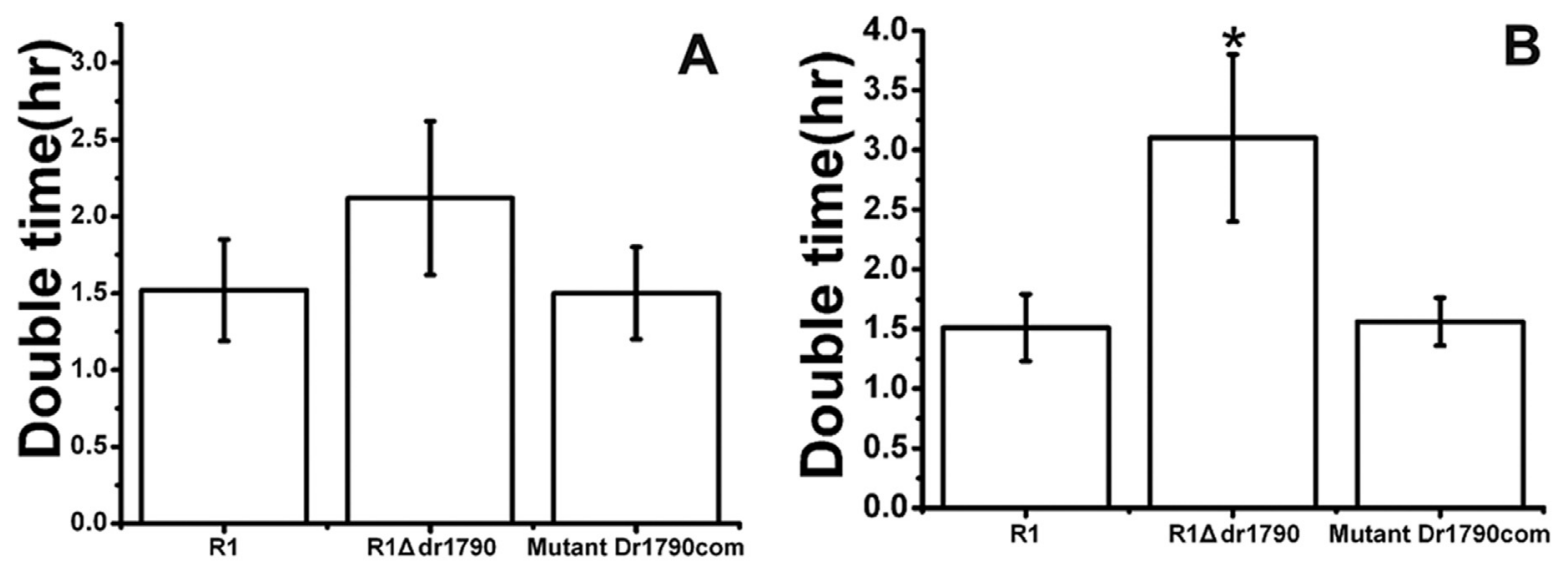

Figure 1 - Growth of wild type $D$. radiodurans $\mathrm{R} 1$ compared with the R1 $\Delta d r 1790$ mutant strain under normal conditions in the $\operatorname{lag}(\mathrm{A})$ and $\log$ (B) phases. The error bars represent the standard deviations from three experiments. 

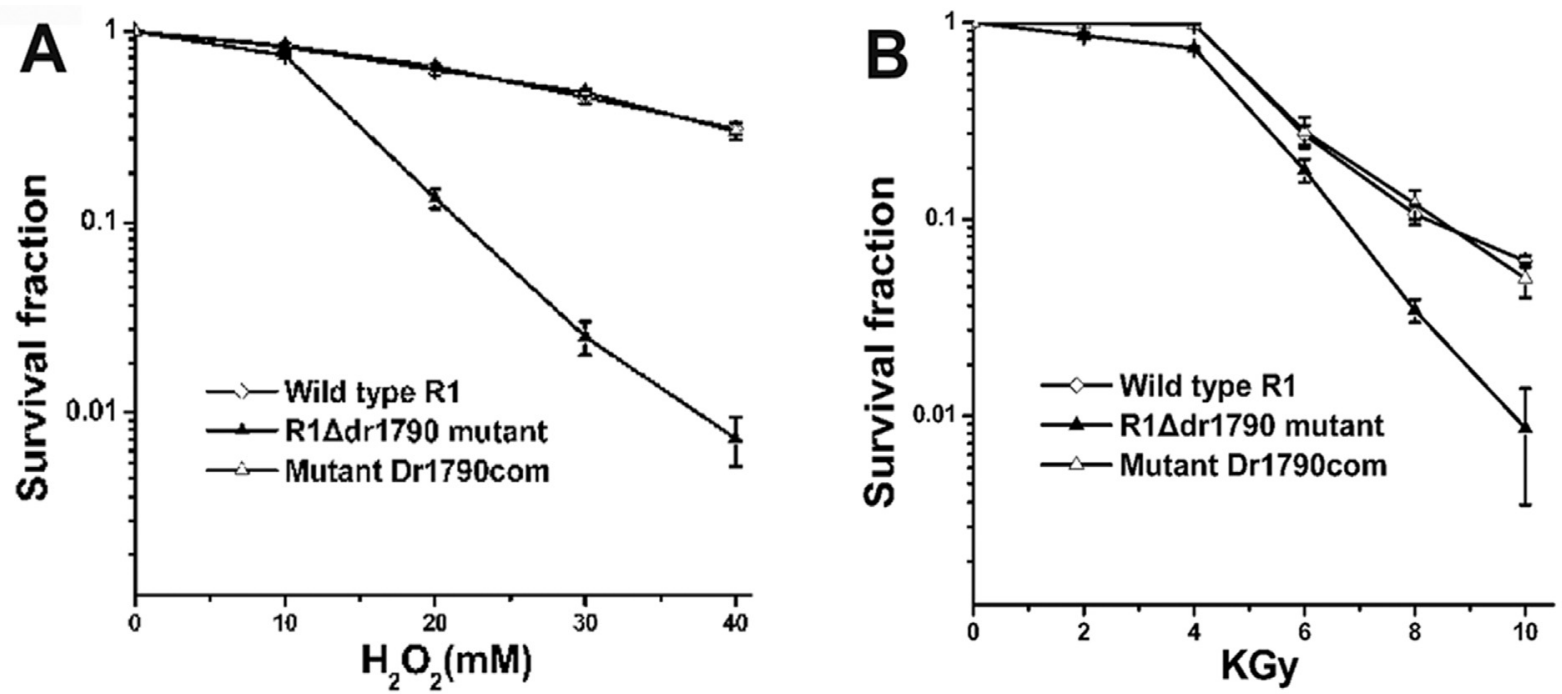

Figure 2 - Survival curves for D. radiodurans following exposure to $\mathrm{H}_{2} \mathrm{O}_{2}(A)$ and gamma radiation (B). Each data point represents the mean of three replicates (bars indicate the standard deviations).

Membrane localization of the DR1790 protein and membrane integrity of the $\mathrm{R} 1 \Delta d r 1790$ mutant strain

Fusion gene expression of the green fluorescence protein (eGFP) gene and $d r 1790$ was performed and analyzed by fluorescence microscopy to confirm the localization of the DR1790 protein (Gao et al., 2008). Figure 4 shows that eGFP-labeled protein (green fluorescence) was localized to the cell membrane; the yellow fluorescence displayed in the merged picture indicates the co-localization of eGFPlabeled proteins and FM4-64 (red fluorescence)-labeled

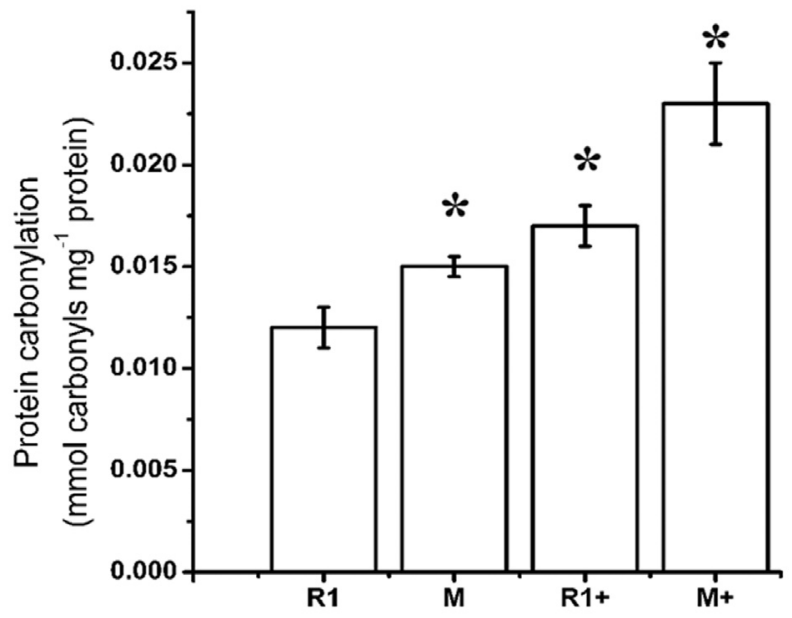

Figure 3 - Comparison of the intracellular protein carbonylation levels between wild type R1 and R1 $\Delta d r 1790$ mutant strains following $\mathrm{H}_{2} \mathrm{O}_{2}$ treatment. R1 and $\mathrm{M}$ represent wild type R1 and R1 $\Delta d r 1790$ mutant strains under normal conditions, respectively. $\mathrm{R} 1+$ and $\mathrm{M}+$ represent wild type $\mathrm{R} 1$ and R1 $\Delta d r 1790$ mutant strains following $\mathrm{H}_{2} \mathrm{O}_{2}$ treatment, respectively. Each data point represents the mean of three replicates (bars indicate the standard deviations). The results were assessed using Student's $t$-test, and statistical significance was considered at $\mathrm{p}<0.05$. membranes, confirming that DR1790 is a membrane protein.

The membrane integrity of the mutant strain was analyzed based on permeability assays using membrane-permeant and membrane-impermeant fluorescence-labeled nucleic acids. The R1 $\Delta d r 1790$ mutant incorporated both the membrane-impermeant dye propidium iodide (PI) and the membrane-permeant dye SYTO9 (Figure 5). Of 1384 mutant bacterial cells counted in 10 different fields, $20 \%$ of the cells incorporated PI (red). In contrast, wild type R1 and complemented mutant Dr1790com strains incorporated SYTO9 (green); however, only 1\% of the cells were PIpositive among the 1464 wild type and 1538 complemented mutant bacteria that were counted in 10 independent fields (Figure 5). Thus, the R1 $\Delta d r 1790$ mutant showed a high proportion of damaged membranes $(20 \%$ red cells observed in the mutant field) compared with wild type and comple-

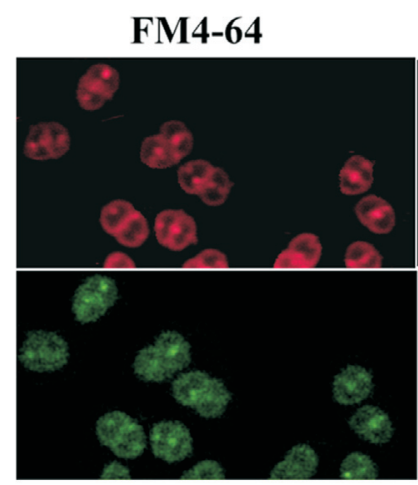

eGFP

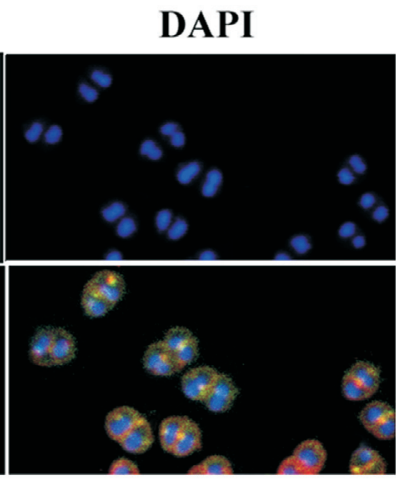

Merge
Figure 4 - Analysis of DR1790 protein localization by fluorescence labeling. Images show FM4-64-stained membranes (red), DAPI-stained DNA (blue), eGFP-labeled proteins (green), and the merged image shows eGFP labeling and FM4-64 and DAPI staining (630). 


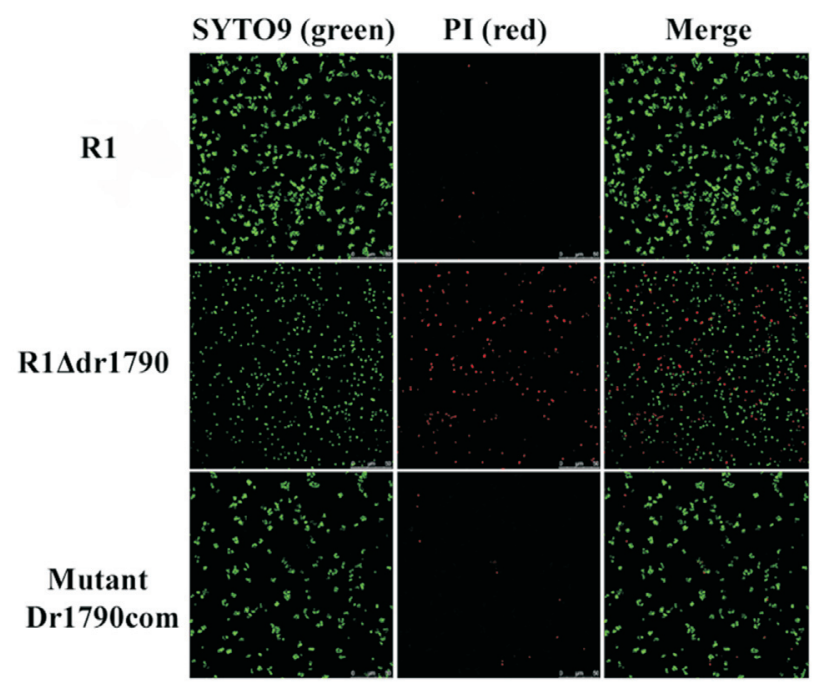

Figure 5 - Stained images of wild type R1, R1 $\triangle d r 1790$ and complemented Mutant Dr1790com strains using a LIVE/DEAD kit (100). Live cells with intact membranes only incorporated SYTO9 (green), whereas dead cells or cells with compromised membranes incorporated PI (red).

mented mutant strains. This result suggests that the DR1790 protein contributes to membrane permeability.

\section{Transcriptional and translational profiles of the DR1790 mutant $v s$. the wild type strain}

2-DE and MS analyses were applied to compare the differential protein expression profiles of the R1 $\Delta d r 1790$ mutant and the wild type R1 strains (Figure 6). Ten protein spots showing two-fold changes in intensity in the $\mathrm{R} 1 \Delta d r 1790$ mutant compared with the wild type R1 were observed, including growth-related metabolism enzymes (IDH, MDH and FBP2), the predicted transmembrane protein transporter DR1909, and the chaperone protein DnaJ (Table 2). The limited information acquired by 2-DE analysis prompted the use of DNA microarray analysis to investigate this issue further.

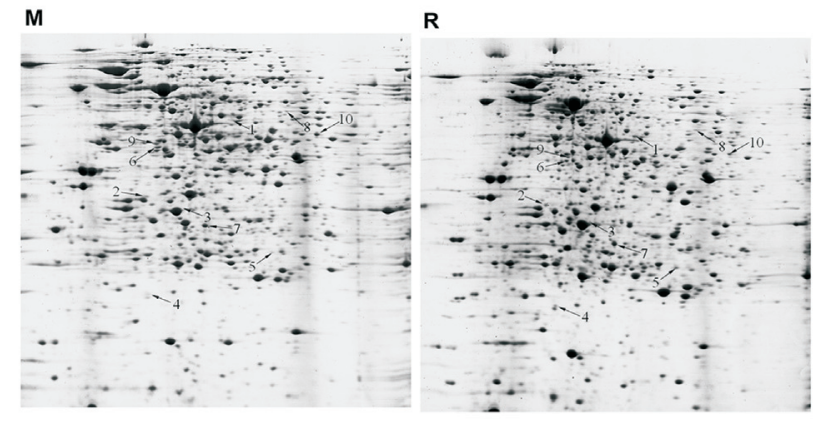

Figure 6 - 2-D gel images visualized by Coomassie Blue staining. The cells were analyzed by 2 -DE and visualized by Coomassie Blue staining as described in the Materials and methods section. (M) R1 $\Delta d r 1790$ mutant, (R) R1.

The transcriptome of the R1 $\Delta$ dr1790 mutant was analyzed and compared with that of the wild type strain under normal growth conditions using oligonucleotide microarray to examine the expression of the entire gene repertoire of $D$. radiodurans in response to $d r 1790$ knockout. In the present study, a two-fold difference in the relative transcription level was selected as the threshold for microarray data analysis as described previously by Chen et al. (2008). We observed that $1.5 \%$ of the genes represented on the microarray $(n=46)$ were differentially transcribed in the R1 $\Delta d r 1790$ mutant compared with the WT. Among these genes, 27 were up-regulated (Table 3), and 19 were downregulated (Table 3). These genes were involved in DNA/RNA repair, energy metabolism, various transporters, proteases and chaperones, stress responses, and translation and transcription functions.

Among the up-regulated genes in the R1 $\Delta d r 1790 \mathrm{mu}-$ tant, three genes were categorized as proteinase genes, six genes were related to protein quality control, and some genes encoded unknown/hypothetical proteins. Similarly, the down-regulated genes in the R1 $\Delta d r 1790$ mutant included four genes that were related to secreted proteins. The effectiveness of the microarray data was further confirmed by real-time quantitative RT-PCR (Table 4). Nota-

Table 2 - Mass spectrometry identification of the protein spots that were separated by 2-DE analysis.

\begin{tabular}{lcclc}
\hline Protein spot & Locus & Length (aa) & Functional category & Expression ratio, mutant 1790/WT (fold) \\
\hline 1 & DR1540 & 430 & Isocitrate dehydrogenase (IDH) & 0.05 \\
2 & DR2013 & 268 & Fructose 1,6-bisphosphatase II (FBP2) & 0.09 \\
3 & DR0325 & 330 & Malate dehydrogenase (MDH) & 0.03 \\
4 & DR1512 & 264 & Elongation factor Ts & 0.09 \\
5 & DR0350 & 571 & Serine/threonine protein kinase & 0.29 \\
6 & DRA0337 & 386 & Glutaryl-CoA dehydrogenase & 2.49 \\
7 & DR1172 & 298 & Cell envelope integrity inner membrane protein & 0.18 \\
8 & DR1909 & 212 & Predicted transmembrane protein transporter & 0.09 \\
9 & DR1148 & 175 & Putative TrkA-C domain protein Tyrosine kinase & 2.56 \\
10 & DR0126 & 312 & Chaperone protein DnaJ & 3.39 \\
\hline
\end{tabular}


Table 3 - Summary of the gene expression results from microarray data. The 27 most highly repressed genes in the R1 $\Delta d r 1790$ mutant. The 19 most highly induced genes in the R1 $\Delta d r 1790$ mutant.

\begin{tabular}{|c|c|c|c|}
\hline Locus & Annotation & Fold decrease & $\mathrm{p}$ value \\
\hline DR1900 & Predicted secreted protein & -23.35015 & $6.9 \mathrm{E}-06$ \\
\hline DRB0006 & Hypothetical protein & -6.61064 & 0.004 \\
\hline DR1702 & $\mathrm{NH} 2$ acetyltransferase & -6.322434 & 0.005 \\
\hline DRB0045 & Hypothetical protein & -5.757131 & 0.005 \\
\hline DR1085 & SAM-dependent methyltransferase & -5.548009 & 0.005 \\
\hline DR0763 & Acetyltransferase & -5.013164 & 0.006 \\
\hline DR1913 & DNA gyrase, subunit A (gyrA) & -4.815689 & 0.011 \\
\hline DR2312 & Carbohydrate kinase, PfkB family & -4.445003 & 0.007 \\
\hline DR1901 & Predicted secreted protein & -3.834828 & 0.019 \\
\hline DR2625 & Lipid A disaccharide synthase-related enzyme & -3.64618 & 0.009 \\
\hline DR2307 & Multidrug-efflux transporter, putative & -3.451829 & 0.010 \\
\hline DR1912 & Protein-tyrosine phosphatase, putative & -3.427269 & 0.011 \\
\hline DR1157 & Hydroxypyruvate reductase, putative & -3.409991 & 0.010 \\
\hline DR2333 & NADH oxidase-related protein & -3.152435 & 0.011 \\
\hline DR1591 & Hypothetical protein & -3.149012 & 0.011 \\
\hline DR1481 & Chlorite dismutase family enzyme & -2.949308 & $4 \mathrm{E}-03$ \\
\hline DR2285 & A-G-specific adenine glycosylase (mutY) & -2.799988 & 0.013 \\
\hline DRC0037 & Nodulation protein-related protein & -2.666197 & 0.015 \\
\hline DRA0300 & Predicted secreted protein & -2.620658 & $4 \mathrm{E}-03$ \\
\hline DR2544 & Predicted secreted protein & -2.604338 & 0.015 \\
\hline DRA0302 & Hypothetical protein & -2.590681 & $2 \mathrm{E}-03$ \\
\hline DR1916 & DNA helicase RecG (recG) & -2.543044 & 0.011 \\
\hline DR1359 & ABC-type metal ion transport system & -2.518423 & 0.060 \\
\hline DR2259 & Transcriptional regulator & -2.45463 & 0.017 \\
\hline DRA0061 & Permease MDR-type & -2.111218 & 0.023 \\
\hline DR0610 & P-loop ATPase of adenylate kinase family & -2.086652 & 0.058 \\
\hline DR2213 & Conserved hypothetical protein & -2.079364 & 0.024 \\
\hline Locus & Annotation & Fold increase & $p$ value \\
\hline DR0888 & Distant homolog of OsmY & 2.02588 & $5 \mathrm{E}-03$ \\
\hline DR2403 & Predicted membrane protein & 2.03289 & 0.025 \\
\hline DR1306 & Predicted secreted protein & 2.03588 & 0.007 \\
\hline DRA0234 & Hypothetical protein & 2.04611 & 0.004 \\
\hline DR1114 & HSP20 & 2.06477 & 0.005 \\
\hline DR0201 & Hypothetical protein & 2.08698 & 0.033 \\
\hline DRA0143 & 3-Hydroxyacyl-CoA dehydrogenase & 2.08777 & 0.024 \\
\hline DR2385 & Phenylacetic acid degradation protein $\mathrm{PaaB}$ & 2.16397 & 0.008 \\
\hline DRA0290 & Cell division protein FtsH (ftsH-3) & 2.21667 & 0.007 \\
\hline DR0607 & GroEL protein (groEL) & 2.28587 & 0.001 \\
\hline DR1046 & ATP-dependent Clp protease, ATP-binding subunit ClpB (clpB) & 2.34619 & 0.012 \\
\hline DR0194 & Predicted Zn-dependent protease & 2.39411 & 0.003 \\
\hline DR0128 & GrpE protein (grpE), HSP20 cofactor & 2.46405 & 0.026 \\
\hline DR0129 & DnaK protein (dnaK) & 2.47161 & $8 \mathrm{E}-03$ \\
\hline DRA0028 & Hypothetical protein & 2.47212 & 0.043 \\
\hline DRA0027 & Putative L-lysine 2,3-aminomutase, Lysine degradation & 2.54777 & 0.016 \\
\hline DR0126 & DnaJ protein (dnaJ-1) & 2.59145 & 0.003 \\
\hline DR1974 & ATP-dependent protease LA (Lon1) & 2.72752 & 0.003 \\
\hline DR0606 & Chaperonin (groES) & 2.76172 & 7E-03 \\
\hline
\end{tabular}


Table 4 - Real-time PCR relative quantification of the expression of repressed and induced genes in the R1 $\Delta d r 1790$ mutant compared with the $D$. radiodurans wild type strain.

\begin{tabular}{lcllc}
\hline Function & Gene name & Locus & Annotation & qRT-PCR Fold change \\
\hline Heat, General & DnaJ-1 & DR0126 & HSP70 cofactor & 2.13 \\
& GrpE & DR0128 & HSP20 chaperonin & 2.45 \\
& DnaK & DR0129 & HSP70 chaperonin & 2.53 \\
& GroES & DR0606 & Hsp10 chaperonin & 2.18 \\
& GroEL & DR0607 & Hsp60 chaperonin & 2.27 \\
& Hsp20 & DR1114 & Molecular chaperone & 4.57 \\
General & HtpX & DR0194 & Zn-dependent protease, Bacillus yugP ortholog & 2.38 \\
& ClpB & DR1046 & ClpB, AAA superfamily ATPase & 31.55 \\
Osmotic & Lon & DR1974 & ATP-dependent Lon protease, bacterial type & 4.68 \\
Others & OsmY & DR0888 & Distant homolog of OsmY & 4.86 \\
& & DR0350 & Serine/threonine protein kinase & -2.14 \\
& & DR1172 & Cell envelope integrity inner membrane protein & -2.32 \\
& & DR1909 & Predicted transmembrane protein transporter & -1.14 \\
\hline
\end{tabular}

bly, many molecular chaperones and proteinases were positively regulated in the R $1 \Delta d r 1790$ mutant, and transporters and kinases were negatively regulated in the $\mathrm{R} 1 \Delta d r 1790$ mutant. These data demonstrate that the deletion of the $d r 1790$ gene significantly increased the amount of misfolded proteins in the cell. Some secreted proteins and transmembrane protein transporters were repressed, indicating that the DR1790 protein could be associated with secretory factors in the membrane.

\section{Discussion}

The extreme resilience of $D$. radiodurans to oxidative and radiation stresses is imparted synergistically by the efficient protection of proteins against oxidative stress and efficient DNA repair mechanisms, enhanced by functional redundancies in both systems (Slade and Radman, 2011). Maleszka et al. (2000) identified an orphan protein (DR1790) in D. radiodurans belonging to the yellowrelated protein family, which was originally identified in Drosophila. A mutation in the yellow-related protein in Drosophila affects the pigmentation of larvae and exerts some effects on insect behavior (Drapeau et al., 2006). In the present study, the predicted yellow-related protein DR1790, which belongs to the ancient Yellow/MRJ protein family, was confirmed to be a membrane-binding protein. A null-mutant strain (R1 $\Delta d r 1790)$ exhibited reduced survival after gamma irradiation and $\mathrm{H}_{2} \mathrm{O}_{2}$ treatment, demonstrating that DR1790 is involved in the radioresistance and antioxidant mechanisms of $D$. radiodurans. Protein, rather than DNA, was suggested to be the principal target of radiation and free radicals, and the degree of cell resistance was determined based on the level of oxidative protein damage (Daly et al., 2007). We observed that the total protein car- bonyl contents increased in the R1 $\Delta d r 1790$ mutant under normal conditions and $\mathrm{H}_{2} \mathrm{O}_{2}$ treatment, demonstrating that the absence of DR1790 increased oxidative damage in cells.

Cellular membranes, which are composed of lipids, proteins, and carbohydrates, are damaged by radiation. The melting of membranes under stress results in permeability barrier loss and leakage, as well as the inability to maintain a proton gradient for respiration. The $D$. radiodurans cell envelope consists of at least five layers (Lancy et al., 1978). D. radiodurans irradiated with $4 \mathrm{kGy}$ loses up to $30 \%$ wet weight resulting from the loss of polysaccharides into the growth medium, which suggests permeability alterations in the cell envelope (Mitchel, 1976). For retaining membrane integrity, D. radiodurans cells were much more resistant to high temperatures when exposed in the dried state as opposed to cells in suspension (Bauermeister et al., 2012). The R1 $\Delta d r 1790$ mutant showed a high proportion of damaged membranes $(20 \%$ red cells observed in the mutant field) compared with wild type and complemented mutant strains. This result suggests that the DR1790 protein contributes to membrane permeability. Consequently, the mutant strain was more sensitive to both ionizing radiation and oxidative stress. However, how DR1790 contributes to bacterial membrane integrity remains unclear. DR1790 may be required for the stability of membrane protein complexes to restore the osmotic imbalance rapidly, and the absence of DR1790 may result in less stability or improperly gated channels or pores. Thus, the isolation of the protein partners of DR1790 may help to clarify the role of this protein in membrane homeostasis. Alterations in membrane integrity may also contribute to the increased sensitivity of $\mathrm{R} 1 \Delta d r 1790$ mutants to oxidative and radiation stresses. 
In the present study, some genes that are involved in protein quality control, such as $d r 1114$ (HSP20), $d r 0129$ (dnaK), dr0126 (dnaJ), dr0607 (groEL), dr1046 (ATP-binding subunit ClpB), and $d r 1974$ (ATP-dependent protease LA, Lon1), were strongly induced in R1 $\Delta d r 1790$ mutants. The induction of these chaperones and proteases suggested that many damaged proteins aggregated in the R1 $\Delta d r 1790$ mutant. Chaperones assist in non-covalent folding or unfolding and in the assembly or disassembly of protein structures in the cell, but do not occur in these structures during the performance of normal biological functions after having completed folding and/or assembly. DnaK/DnaJ/GrpE and GroEL/ES are the two primary chaperone foldase systems in prokaryotic cells (Hoffmann et al., 2004). ATP-dependent proteases function in protein processing and play an essential role in diverse stress responses (Gottesman, 2003). In D. radiodurans, the majority of cellular proteolysis is performed by ATP-dependent proteases that belong to the Lon (Lon1 and Lon2) and Clp families (ClpA, ClpB, ClpC, ClpX and ClpP). The ClpPX protease is required for radioresistance and regulates cell division after $\gamma$-irradiation in D. Radiodurans (Servant $e t$ al., 2007). ClpB from Myxococcus xanthus functions as a chaperone protein and plays an important role in cellular heat and osmotic stress tolerance mechanisms during both vegetative growth and development (Pan et al., 2012). $\mathrm{ClpB}$ and the DnaK system act synergistically to remodel proteins and to dissolve aggregates (Doyle et al., 2007). HSPs function as molecular chaperones that prevent protein denaturation and aggregation (Feder and Hofmann, 1999; Matuszewska et al., 2008). Additionally, some genes that are involved in protein secretion and transport are strongly suppressed in R1 $\Delta d r 1790$ mutants, such as secreted proteins (DR1900, DR1901, DRA0300, and DR2544) and transmembrane transporter proteins (DR1909), indicating that the DR 1790 protein could be related to secretory factors in the membrane. D. radiodurans contains many secreted proteases and transporters that provide exogenous amino acids as protein building blocks and peptides as components of manganese complexes (Slade and Radman, 2011). After irradiation in D. radiodurans, 10 secreted subtilisin-like proteases, and 4 peptide and amino acid $\mathrm{ABC}$ transporters were highly induced (Makarova et al., 2000; Ghosal et al., 2005). Thus, the low growth rate and sensitivity to hydrogen peroxide and radiation in the $\mathrm{R} 1 \Delta d r 1790$ mutant were closely associated with the induction of these chaperones and proteases and with the suppression of secreted and transported proteins. Additionally, the expression of some genes involved in antioxidant systems, electron transport, and energy metabolism were also significantly altered by the disruption of DR1790.

In conclusion, we presented the first experimental evidence that a protein from the ancient yellow protein family plays a role in the survival of prokaryote cells during a damage response. The DR1790 protein from the ancient yellow protein family plays a pleiotropic role in the survival of prokaryotic cells and contributes to the extraordinary resistance of $D$. radiodurans against oxidative and radiation stresses. Further studies are required to understand the mechanisms of the action that are mediated by DR1790 during this process and to identify critical protein interactions.

\section{Acknowledgments}

This work was supported by grants from National Natural Science Foundation of China (31370102), the project for genetically modified organisms breeding from the Ministry of Agriculture of China (2014ZX08009-003), a grant from Special Fund for Agro-scientific Research in the Public Interest from the Ministry of Agriculture of China (201103007), the Natural Science Foundation of Zhejiang Province (LY13C010001), the Public Project of Zhejiang Province (2014C33024), the Fundamental Research Funds for the Central Universities from Zhejiang University (2012FZA6014), and the Project for Zhejiang Provincial Construction of Technology Innovation Team (2010R50033).

\section{References}

Battista JR (1997) Against all odds: the survival strategies of Deinococcus radiodurans. Annu Rev Microbiol 51:203224.

Battista JR (2000) Radiation resistance: the fragments that remain. Curr Biol 10:204-205.

Bauermeister A, Hahn C, Rettberg P et al. (2012) Roles of DNA repair and membrane integrity in heat resistance of Deinococcus radiodurans. Arch Microbiol 194:959-966.

Brim H, McFarlan SC, Fredrickson JK et al. (2000) Engineering Deinococcus radiodurans for metal remediation in radioactive mixed waste environments. Nat Biotechnol 18:85-90.

Chen H, Xu G, Zhao Y et al. (2008) A Novel OxyR Sensor and Regulator of Hydrogen Peroxide Stress with One Cysteine Residue in Deinococcus radiodurans. PLoS One 3:e1602.

Daly MJ, Gaidamakova EK, Matrosova VY et al. (2007) Protein oxidation implicated as the primary determinant of bacterial radioresistance. PLoS Biol 5:e92.

Drapeau MD, Albert S, Kucharski R et al. (2006) Evolution of the Yellow/Major Royal Jelly Protein family and the emergence of social behavior in honey bees. Genome Res 11:13851394.

Drapeau MD (2003) A novel hypothesis on the biochemical role of the Drosophila Yellow protein. Biochem Biophys Res Commun 311:1-3.

Doyle SM, Hoskins JR, Wickner S (2007) Collaboration between the ClpB AAA+ remodeling protein and the DnaK chaperone system. Proc Natl Acad Sci USA 104:11138-11144.

Feder ME, Hofmann GE (1999) Heat shock proteins, molecular chaperones, and the stress response. Annu Rev Physiol 61:243-282.

Ferguson LC, Green J, Surridge A et al. (2011) Evolution of the Insect Yellow Gene Family. Mol Biol Evol 28:257-272.

Gao G, Lu H, Huang L et al. (2005) Construction of DNA damage response gene pprI function-deficient and function-com- 
plementary mutants in Deinococcus radiodurans. Chinese Sci Bull 50:311-316.

Gao G, Fan L, Lu H et al. (2008) Engineering Deinococcus radiodurans into biosensor to monitor radioactivity and genotoxicity in environment. Chinese Sci Bull 53:16751681 .

Ghosal D, Omelchenko MV, Gaidamakova EK et al. (2005) How radiation kills cells: survival of Deinococcus radiodurans and Shewanella oneidensis under oxidative stress. FEMS Microbiol Rev 29:361-375.

Gottesman S (2003) Proteolysis in bacterial regulatory circuits. Annu Rev Cell Dev Biol 19:565-587.

Hoffmann JH, Linke K, Graf PCF et al. (2004) Identification of a redox-regulated chaperone network. EMBO J 23:160-168.

Lancy PJ, Murray RG (1978) The envelope of Micrococcus radiodurans: isolation, purification, and preliminary analysis of the wall layers. Can J Microbiol 24:162-176.

Lange CC, Wackett LP, Minton KW et al. (1998) Engineering a recombinant Deinococcus radiodurans for organopollutant degradation in radioactive mixed waste environments. Nat Biotechnol 16:929-933.

Lu H, Gao G, Xu G et al. (2009) Deinococcus radiodurans PprI switches on DNA damage response and cellular survival networks after radiation damage. Mol Cell Proteomics 8:481-494.

Makarova KS, Aravind L, Wolf YI et al. (2001) Genome of the extremely radiation-resistant bacterium Deinococcus radiodurans viewed from the perspective of comparative genomics. Microbiol Mol Biol Rev 65:44-79.

Makarova KS, Aravind L, Daly MJ et al. (2000) Specific expansion of protein families in the radioresistant bacterium Deinococcus radiodurans. Genetica 108:25-34.

Maleszka R, Kucharski R (2000) Analysis of Drosophila yellow-B cDNA reveals a new family of proteins related to the royal jelly proteins in the honeybee and to an orphan protein in an unusual bacterium Deinococcus radiodurans. Biochem Biophys Res Commun 270:773-776.

Markillie LM, Varnum SM, Hradecky P et al. (1999) Targeted mutagenesis by duplication insertion in the radioresistant bacterium Deinococcus radiodurans: radiation sensitivities of catalase $(\mathrm{kat} A)$ and superoxide dismutase $(\operatorname{sod} A)$ mutants. J Bacteriol 181:666-669.

Mattimore V, Udupa KS, Berne GA et al. (1995) Genetic characterization of forty ionizing radiation-sensitive strains of Deinococcus radiodurans: linkage information from transformation. J Bacteriol 177:5232-5237.

Matuszewska E, Kwiatkowska J, Kuczynska-Wisnik D et al. (2008) Escherichia coli heat-shock proteins IbpA/B are involved in resistance to oxidative stress induced by copper. Microbiol 154:1739-1747.
Minton KW (1994) DNA repair in the extremely radioresistant bacterium Deinococcus radiodurans. Mol Microbiol 13:915.

Mitchel RE (1976) Ionizing radiation damage in Micrococcus radiodurans cell wall: release of polysaccharide. Radiat Res 66:158-169.

Pan J, Wang J, Zhou Z et al. (2009) IrrE, a global regulator of extreme radiation resistance in Deinococcus radiodurans, enhances salt tolerance in Escherichia coli and Brassica napus. PLoS One 2:e4422.

Pan H, Luan J, He X et al. (2012) The clpB gene is involved in the stress response of Myxococcus xanthus during vegetative growth and development. Microbiol 158:2336-2343.

Servant P, Jolivet E, Bentchikou E et al. (2007) The ClpPX protease is required for radioresistance and regulates cell division after gamma-irradiation in Deinococcus radiodurans. Mol Microbiol 66:1231-1239.

Shu H, Tian B (2010) Radioresistance analysis of Deinococcus radiodurans gene DR1709 in Escherichia coli. Afr J Microbiol Res13:1412-1418.

Shu H, Tian B (2012) The radioresistance analysis of the Deinococcus radiodurans gene DR1709 in human bone marrow cell line KG1a. Afr J Microbiol Res 6:40-45.

Slade D, Lindner AB, Paul G et al. (2009) Recombination and replication in DNA repair of heavily irradiated Deinococcus radiodurans. Cell 136:1044-1055.

Slade D, Radman M (2011) Oxidative Stress Resistance in Deinococcus radiodurans. Mol Biol Rev 75:133-191

Tian B, Sun Z, Shen S et al. (2009) Effects of carotenoids from Deinococcus radiodurans on protein oxidation. Lett Appl Microbiol 49:689-694.

Wang P, Schellhorn HE (1995) Induction of resistance to hydrogen peroxide and radiation in Deinococcus radiodurans. Can J Microbiol 41:170-176.

Wang L, Xu G, Chen H et al. (2008) DrRRA: a novel response regulator essential for the extreme radioresistance of Deinococcus radiodurans. Mol Microbiol 67:1211-1222.

Wettenhall JM, Smyth GK (2004) limmaGUI: a graphical user interface for linear modeling of microarray data. Bioinformatics 20:3705-3706.

Wu Y, Chen W, Zhao Y et al. (2009) Involvement of RecG in $\mathrm{H}_{2} \mathrm{O}_{2}$-induced damage repair in Deinococcus radiodurans. Can J Microbiol 55:841-848.

Xu G, Wang L, Chen $\mathrm{H}$ et al. (2008) RecO is essential for DNA damage repair in Deinococcus radiodurans. J Bacteriol 190:2624-2628.

Associate Editor: Luis Henrique Souza Guimarães

All the content of the journal, except where otherwise noted, is licensed under a Creative Commons License CC BY-NC. 